\title{
Audiences' experience of proximity and co-presence in live dance performance
}

Corinne Jola and Matthew Reason

This is an Accepted Manuscript of a book chapter published by Bloomsbury Academic in Theatre and cognitive neuroscience on 25th February 2016, available online: https://www.bloomsbury.com/uk/theatre-andcognitive-neuroscience-9781472584809/ 
Audiences’ Experience of Proximity and Co-Presence in Live Dance Performance Corinne Jola and Matthew Reason

\section{Introduction}

Spectators' experiences in watching dance have recently gained much attention in scientific research. Cognitive neuroscience, a field that generally studies the neuronal processes linked with sensory stimulation, action execution, memory, language, attention, emotion and consciousness, had over the last ten years sought to better understand how the human brain responds to watching dance movements. For example, neuroscientific publications have dealt with the neuronal responses of expert or novice spectators watching dance. ${ }^{1}$

The majority of these studies are based on the theory of mirror neurons, brain cells often described as forming a mirror neuron network. The brain areas containing these cells showed functional relevance for action execution, such as dancing, but also for passive observation. ${ }^{2}$ This overlap of brain activity during passive action observation and action execution has been explained in terms of a 'mirroring' mechanism, an internal, sub-threshold neuronal resonance of the movements that are seen as if the spectator him/herself was moving. To some extent, the findings of this research capture the neural basis of the ephemeral experience of watching dance. However,

\footnotetext{
${ }^{1}$ See Bettina Bläsing, Beatriz Calvo-Merino, Emily S. Cross, Corinne Jola, Juliane Honisch, Catherine J. Stevens, 'Neurocognitive Control in Dance Perception and Performance, Acta Psychologica (Amst) 139(2) (2012); Vassilis Sevdalis and Peter E. Keller, 'Captured by Motion: Dance, Action Understanding, and Social Cognition', Brain and Cognition 77(2) (2011).

${ }^{2}$ For an overview of the brain areas found to be relevant in passive dance observation, see Julia F. Christensen and Beatriz Calvo-Merino, 'Dance as a Subject for Empirical Aesthetics', Psychology of Aesthetics Creativity and the Arts
} SI, 7(1) (2013). 
there are limitations to such studies, including - and most relevant to this paper - the technological challenges accompanying research on audience responses to live performance.

Due to the constraints of brain imaging technologies, and the requirement to fulfil the scientific principles of reliability and reproducibility, scientists generally employ digital displays (e.g. images, video recordings) for all types of experiments that require visual perception. While this approach has enhanced our understanding of the complex neuronal processes of perception and cognition, as a research approach into the responses of spectators to dance, it neglects numerous possible experiential differences between watching dance in real live and on mediated platforms (e.g. video). Such differences include the social experience of going to the theatre and the sense of expectation (or eventness) that may develop in a live setting. Here, we focus on another perhaps more elusive difference, which is the impact of the dancer's live 'presence' on the spectators' experience. While a video recording is able to capture a performance in terms of its appearance in time and space, and represent this to us, there remains the frequently articulated feeling that something is lost in the process, that loss being the presence of the performer.

This paper thus explores the impact of the dancer's live presence on the spectators' neuronal responses. To this end, we shall firstly articulate our understanding of presence, drawing on theoretical literature from the performing arts (2.), then we shall engage with empirical research on the effects performer proximity and co-presence have on the experiences of the audience. At the outset, we acknowledge the limitations of our discussion, including the focus on an existing and already published study that was based on a particular set of circumstances ${ }^{3}$ also described in more detail below (3.). However, we hope that by beginning to think about presence - central to artistic discourses about performance - as a modulating factor in action observation, we can place presence also within a scientific discourse on spectatorial experiences of dance.

\footnotetext{
${ }^{3}$ Corinne Jola, Ali Abedian-Amiri, Annapoorna Kuppuswamy, Frank E. Pollick, Marie-Hélène Grosbras, 'Motor Simulation Without Motor Expertise: Enhanced Corticospinal Excitability in Visually Experienced Dance Spectators',
} PLoS ONE 7(3) (2012). 


\section{Defining presence, questioning presence}

In Oxford English Dictionary, the etymology of 'presence' is rooted in the Latin praesentia: 'being at hand'. There are two ideas that are central to the concept. The most obvious is one of 'proximity' in space and simultaneity in time: being present in the here and the now. The other notion is that presence has a relational component. 'Being at hand' implies a dynamic relationship between two factors, which in terms of dance becomes the 'co-presence' between spectators and performers. A third meaning of presence is not derived from the above etymological analysis but provided in the same Oxford English Dictionary in a further definition: 'The impressive manner or appearance of a person: Richard was not a big man but his presence was overwhelming.' Such a description of 'stage presence', what might also be called charisma and associated with 'star' power, is an important concept, but one that we do not develop further in this paper.

Presence as discussed here is therefore associated with the temporal and spatial relationship between spectator and performer. As such, it is directly linked to the main elements of live performance, which Wurtzler describes as 'characterized by spatial co-presence and temporal simultaneity of audience and event'. ${ }^{4}$ For some performance scholars this quality of presence has an ontological importance, with one example being Beckerman's assertion of the difference between watching action on screen and on stage: 'Eliminate the actuality of man and eliminate theatre. The experience of seeing human beings battle time and space cannot be the same as seeing visual images upon a screen'. ${ }^{5}$ If we translate such attitudes from the theatre to dance we have a sense that something is lost in the process of even the most faithful recording.

More than simply occupying the same time and space as each other, co-presence implies a kind of mutuality, that is an awareness or active going between performer and recipient. Stanton

\footnotetext{
${ }^{4}$ Steve Wurtzler, “'She Sang Live, but the Microphone was Turned Off”: The Live, the Recorded and the Subject of Representation', in R. Altman (ed.), Sound Theory Sound Practice (London: Routledge, 1992), p. 89.

${ }^{5}$ Bernard Beckerman, Dynamics of Drama (New York: Drama Book Specialists, 1979), p. 7.
} 
Garner, for example, notes that it is not only the audience that is looking, but that the gaze of the spectator is returned, the performers looking at the audience and asserting their subjectivity. ${ }^{6}$ Similarly, Erika Fischer-Lichte describes how 'performances are generated and determined by a self-referential and ever-changing feedback loop'. ${ }^{7}$ This description is again of a co-presence, a mutual relationship between watched and watcher than impacts upon both.

Such descriptions have been questioned. In writing about audiences' perceptions of liveness across different art forms and media, Barker, for example, puts an emphasis on co-presence as 'the sine qua non for theatre and performance studies'. Barker suggests that an important element in this relationship is that of 'experienced risk', in the sense that the performance is not "locked" and might be shaped by the audience responses'. However, Barker dismisses this as a 'strange notion', pointing out that audiences typically see a performance only once and are therefore unable to truly know in what ways it might have changed in their presence alone. ${ }^{8}$

Meanwhile, in his book Liveness, Auslander pointed out the ways in which mediatization (from recordings to large screen relays to live broadcasts to amplification) complicates the apparent essentialism of performer-spectator relationship. Most particularly Auslander challenges the 'unreflective assumptions' that he perceived as underpinning value judgements based on liveness. ${ }^{9}$ In relation to ideas of presence, these 'unreflective assumptions' might include ideas of an 'energy that supposedly exists between performers and spectators', that live performance automatically establishes a 'community' either within the audience or between spectators and performers, and that of a cultural value or authenticity that is associated with having been at or in the presence of the

\footnotetext{
${ }^{6}$ Stanton Garner, Bodied Spaces: Phenomenology and Performance in Contemporary Drama (Ithaca, NY: Cornell University Press, 1994), p. 48.

${ }^{7}$ Erika Fischer-Lichte, The Transformative Power of Performance: A New Aesthetics (Abingdon and New York: Routledge, 2008), p. 38.

${ }^{8}$ See Martin Barker, Live to Your Local Cinema: The Remarkable Rise of Livecasting (Basingstoke: Palgrave Macmillan, 2013), pp. 57 and 44.

${ }^{9}$ Philip Auslander, Liveness: Performance in a Mediatized Culture (London and New York: Routledge, 1994), p. 2.
} 
performer. Elsewhere, Auslander questions the assumption that 'the presence of the actor as one living human being before others is spiritually and psychologically liberating in itself'. ${ }^{10}$

At a theoretical level the importance and impact of audience-performer co-presence is therefore contested. Additionally, scientists are more inclined to position forms of presence that go beyond the pure sharing of time and space between two people as something metaphysical that would resist empirical investigation. Moreover, a study based on stimuli that, as Barker ${ }^{11}$ puts it, are not 'looked at' and shaped by the spectators, causes concern for the reliability of scientific measures. $^{12}$

One of the objectives of this paper is thus to explore how these ideas of presence are manifested experientially amongst audiences and whether we can empirically investigate the psychological effects of these experiences as well as the neuronal basis of presence when watching dance. As indicated above, discourses around presence are overlapping and often in conflict. Presence might be articulated in terms of simple temporal and spatial co-existence (proximity) or as a mutual reciprocity between performer and audience (co-presence). Interestingly, Copeland presents what he describes as a 'litmus test' for presence, suggesting that 'perhaps being 'in the presence of' a performer means that we could, if we so desired, reach out and touch (that) someone'. ${ }^{13}$ Here presence becomes direct and unmediated proximity and it was this most absolute form of presence that we sought to investigate here.

\section{Dataset}

\footnotetext{
${ }^{10}$ Ibid., p. 37.

${ }^{11}$ Barker, Live to Your Local Cinema.

${ }^{12}$ See also Corinne Jola and Marie-Hélène Grosbras, 'In the Here and Now: Enhanced Motor Corticospinal Excitability in Novices when Watching Live Compared to Video Recorded Dance', Cognitive Neuroscience 4(2) (2013).

${ }^{13}$ Roger Copeland, 'The Presence of Mediation', The Drama Review 34(4) (1990), p. 34.
} 
The dataset utilized here was taken from a published study that investigated how the visual experience of spectators modulates their neuronal activity in the form of sensorimotor resonance when watching dance. ${ }^{14}$ Spectators with experience in watching ballet, Indian dance or no visual experience watched three types of solo performances: ballet (concatenated solo parts from a classical version of the 'Sleeping Beauty'), Bharatanatyam (a classical Indian dance performance and henceforth referred to as Indian dance) and a miming act (consisting of non-verbal actions originally employed as a non-dance control condition and henceforth referred to as acting control condition). Notably, the different solos were all performed for the same duration (five minutes), using the same spatial extensions in a dance studio (6.8 $\mathrm{m}$ in width and $13.5 \mathrm{~m}$ in length). At points, the performers were at close quarters - in a context where the participants could, although none did, 'reach out and touch' the performer.

The fact that the spectators' neuronal responses were probed in situ, while they were watching the live performances, is uncommon for such a study. Moreover, participants' subjective experiences were also assessed by means of a qualitative audience interview immediately after the performances. Further, a bundle of post-hoc questionnaires assessed spectators' individual characteristics, such as their empathic abilities by means of the Autistic Questionnaire ${ }^{15}$ and the Interpersonal Relationship Index, ${ }^{16}$ and their enjoyment of watching the solos by means of rating scores from 1 (did not enjoy it at all) to 10 (enjoyed it very much) for each individual performance. Their age and gender were also assessed.

\footnotetext{
${ }^{14}$ Corinne Jola, Ali Abedian-Amiri, Annapoorna Kuppuswamy, Frank E. Pollick, Marie-Hélène Grosbras, 'Motor Simulation Without Motor Expertise: Enhanced Corticospinal Excitability in Visually Experienced Dance Spectators', PLoS ONE 7(3) (2012).

15 AQ; Simon Baron-Cohen, Sally Wheelwright, Richard Skinner, Joanne Martin, Emma Clubley, 'The Autism Spectrum Quotient (AQ): Evidence from Asperger Syndrome/High Functioning Autism, Males and Females, Scientists and Mathematicians', Journal of Autism and Developmental Disorders 31(5-17) (2001).

${ }^{16}$ IRI; Marc H. Davis, ‘A Multidimensional Approach to Individual Differences in Empathy', JSAS Catalog of Selected Documents in Psychology 10(85) (1980).
} 
Spectators' neuronal responses were measured indirectly by externally stimulating the hand and forearm area of their motor cortex and measuring the motor evoked potential that the stimulation evoked. ${ }^{17}$ The more the cortex is engaged in a movement (e.g. preparation of an action, observation of a movement), the higher its excitability, and the higher the excitability, the lower the threshold to trigger an action potential. Thus, if the passive spectator is mentally fully drawn into the dance movements observed, the larger the motor evoked potentials in the arm and hand muscle groups will be.

The qualitative interviews had a semi-structured format, with one specific prompt asking participants how they felt the circumstances of the performance impacted on their experience. The material gathered from these responses was then grouped thematically and analysed in the light of the a priori theoretical conceptions of presence described above. Notably, we tested neuronal responses in a live setting in an attempt to match the spectators' visual experience. However, 'liveness' in itself was not investigated in 2012, neither has it received attention in the form of an experience in later studies. ${ }^{18}$

\section{Qualitative interviews}

The discussion of the spectators' responses in this section is divided into the themes of 'proximity' and 'co-presence', as identified above. Within these themes, the nature of spectators' responses had diverse expressions: these include differences in the value placed on the experience; difference in emotional responses; and differences in the description of the audience/performer relationship.

\footnotetext{
${ }^{17}$ E.g. Eric Wassermann, Charles Epstein, Ulf Ziemann, Oxford Handbook of Transcranial Stimulation (Oxford: Oxford University Press, 2008).

${ }^{18}$ Jola and Grosbras, 'In the Here and Now'.
} 


\subsection{Proximity}

The least complex responses articulated the benefits of being closer to the performer in terms of being able to see more and see better. 'I could see exactly what they were doing' said one spectator. For some this included the ability to see particular details of the costume, which produced both positive (appreciative of the detail and precision) and negative responses (one spectator felt the fabric of the ballet dancers' dress would have looked better from a distance).

However, the predominant benefit of proximity in this context was the greater access it gave to the performer's face. Spectators reported this in terms of being able to see facial expressions and the emotions conveyed through the eyes. What is crucial here is the centrality of the face to establishing an empathetic relationship with the performer, as one spectator remarked 'I really enjoyed being that close to a person's thoughts and emotions.' While another commented: 'You can see more of her facial expressions, her emotions, there were some smiles and some, you know, just basically the dancer's emotions, so you were able to connect more and feel more of her movement.' It is worth noting that these examples are one-directional, describing the spectator's gaze upon the performer.

The audience contrasted this proximity to the experience of distance in a large theatre setting and compared it to the experience of film and television. If considered purely in these terms, then closeness simply enables live dance to replicate the access to facial expressions, which constitutes a primary element in the experience of film and television. Indeed, for some spectators this was the extent of their articulation. Proximity in this sense, therefore, might allow greater empathy with the performer, but was not connected to anything particular to the live performance or to a sense of copresence.

\subsection{Co-presence}

However, presence impacted other participants in additional ways that can be discussed in terms of co-presence. Crucially, here it was not just the performer who was close to the spectator - which is 
the experience of watching a close-up on screen - but a shared moment, a mutual closeness in which the spectator was also close to the performer.

Again, it is worth stressing that this elicited a diversity of responses, both in terms of what was noticed and the value that was placed upon it. For some spectators mutual proximity results in the sense of having established a direct and reciprocal relationship with the performer. 'I was responding to the way she was smiling and the way she was looking' said one spectator, 'because of the smile, she made you want to smile.' Another noted that she felt the performer was 'asking me to smile about something... I felt I had to smile'. There is a mutual presence here, a reciprocity that can be thought of in terms of an empathetic, face-to-face connection. For these spectators who related proximity to reciprocity the experience was intense, intimate, real and, as a result, pleasurable and enhanced.

For other spectators these same elements - mutual presence and reciprocity - produced very different, negative responses. In some instances this was because the intensity was too much or because the sense of being mutually involved was uncomfortable. One commented, 'you do feel a bit self-conscious when the dancer's performing just for you', another that 'I was almost too scared to look at her face and it was more like look at her feet and look at the bottom of the dress... I did think, no I don't want to look her in the eye.' Another that: 'They came up quite close, which was quite quite weird, and they looked you in the eye as well [which felt] a bit disconcerting. Like you're the audience I guess, they shouldn't be, you should focus on them rather than them focus on you sort of thing.'

The self-consciousness that such mutual presence elicits produces a sense of involvement in the performance, which is experienced both positively and negatively, and which is in clear contrast to the spectators' sense of invisibility when seated in the collective audience of a theatre or watching film or television.

Notably, for some spectators the combination of closeness and co-presence resulted in a particular kind of specialness in the experience. For many this was articulated as the sensation that 
the performer was dancing just for them: 'It was like performed just for me', said one spectator; 'they were performing for me', said another; 'it almost felt like they were doing it just for me'. For those spectators with a particular disposition (habitus) to a particular form of dance, this produced a particularly intense, emotional response. This was mainly the case with ballet, as illustrated by this particular spectator: 'the fact she was doing it for me, you know, it was wonderful, I just felt so special, oh I'll cry [laughs]'.

\section{Quantitative analyses}

\subsection{Experiencing presence}

Of the twenty-nine participants (eighteen female), all but ten spectators (five female) made some form of statements about presence (proximity, reciprocity, specialness) in relation to the live solo performances seen. We then grouped all the statements in the two categories - proximity and copresence - outlined above.

Presence was described as a neutral experience in only four instances, which were thus collated with the cases that did not discuss presence. We argue that the spectators who did not mention presence, did not experience it as something special, but rather neutrally. We therefore refer to the group as 'N/A', when no information of presence was given or when presence was experienced as neutral.

\begin{tabular}{|c|c|c|c|c|c|c|}
\hline & & Positive & Negative & Neutral & Total & $\begin{array}{l}\text { Not } \\
\text { Mentioned }\end{array}$ \\
\hline & Male & 2 & 3 & 2 & 7 & \\
\hline & Female & 8 & 2 & 0 & 10 & \\
\hline & Total & 10 & 5 & 2 & 17 & 12 \\
\hline & Male & 4 & 2 & 1 & 7 & \\
\hline & Female & 4 & 3 & 1 & 8 & \\
\hline & Total & 8 & 5 & 2 & 15 & 14 \\
\hline Overall & & 18 & 10 & 4 & 30 & $10 *$ \\
\hline
\end{tabular}


Table 1. Distribution of audiences' $(\mathrm{N}=29)$ evaluations of proximity and co-presence. $10 *$ refers to the total number of spectators who did not mention presence at all in relation to these performances (i.e. neither proximity nor co-presence).

As can be seen from

Table 1 , there were more positive than negative responses to proximity compared to copresence. The number of times proximity or co-presence was mentioned with a positive, negative, or N/A connotation was however equally distributed [Chi-square, $\mathrm{df}(4)=5.453, p=0.244]$. Also, the Pearson chi-square showed no significant gender differences regarding the valence of the responses, neither for proximity $[p=0.668]$ nor closeness $[p=0.280]$. Notably, and this is also true for all of the following analyses, the number of observations is very small. Hence, while it is possible that some tendencies would reach significance with a larger sample size and/or if spectators were asked to give a response to each type of presence by means of a questionnaire, one should also consider that these observations stem from previously analysed data. In addition, due to multiple testing, a higher significance threshold should be considered.

Seven spectators specifically linked notions of presence to only one of the three performance types. In total, $75 \%$ of experienced ballet spectators and $78 \%$ of the novice group but only $38 \%$ of Indian dance spectators talked about their experience of presence. However, the different proportions do not deviate significantly from an equal distribution $[p=0.145]$. The chi-square showed a very strong trend for more positive than negative proximity notions for ballet dancers (6 vs 1 ) compared to novices $(2 \mathrm{vs} 4 ;[\mathrm{df}(1)=3.856, p=0.053]) .{ }^{19}$ No such trend was observed for copresence $[p=0.221]$.

\footnotetext{
${ }^{19} p$-values $\leq 0.050$ are considered as significant. Due to the small sample size, we also report $p$-values that show a $p$ value 'near' statistical significance (see for example N. A. Desbiens, 'A Novel Use for the Word "Trend" in the Clinical Trial Literature', American Journal of the Medical Sciences 326(2) (2003). We defined p-values between 0.050 and $\leq 0.060$ as a 'very strong trend', $p$-values $>0.060$ and $\leq 0.080$ as a strong trend, and $p$-values $>0.080$ and $<0.110$ as indicating a 'trend'. Anything $>0.110$ is considered non-significant. Clearly, we should investigate our preliminary
} 
We then investigated whether individual characteristics such as age, enjoyment, or empathy were affected by notions of presence (positive-negative-N/A) using univariate ANOVAs. Age was not significant for neither proximity $[p=0.156]$ nor co-presence experiences $[p=0.941]$, and was thus not discussed further.

However, spectators' average enjoyment across the three solos depended on their evaluation of the experience of presence $[\mathrm{F}(2,28)=4.637, p=0.019]$. Spectators who reported proximity as a negative experience $[5.07 \pm 1.75],{ }^{20}$ enjoyed the performances significantly less $[p=0.028]$ than spectators who reported proximity as a positive experience [7.30 \pm 1.78$]$ or spectators who did not report proximity as either positive or negative [7.19 $\pm 1.04 ; p=0.028]$. Furthermore, enjoyment ratings for the individual performances led to significantly different evaluations of proximity for Indian dance $[\mathrm{F}(2,28)=4.500, p=0.021]$ and did show a trend for ballet $[\mathrm{F}(2,28)=2.776, p=0.081]$. Descriptive data and the post-hoc $t$-test for the effects of the proximity experiences on individual enjoyment ratings are shown in Figure 1.

Co-presence statements showed a very strong trend toward a significant effect on the average enjoyment ratings $[\mathrm{F}(2,28)=3.208, p=0.057]$. Spectators who experienced co-presence negatively exhibited a very strong trend of significantly lower enjoyment ratings [5.33 \pm 1.58$]$ compared to spectators who did not mention co-presence in the interviews $[7.29 \pm 1.09 ; p=0.054]$ ). However, positive co-presence experiences across the three performance styles was not significantly different from either negative or neutral experiences $[6.96 \pm 2.13 ; p \geq 0.213]$. For the performance-specific enjoyment ratings, we observed a very strong trend toward the significant effect of co-presence on the acting control condition $[\mathrm{F}(2,28)=1.886, p=0.053]$ and a trend toward a significant effect on enjoyment ratings of ballet $[\mathrm{F}(2,28)=2,574, p=0.095]$. See Figure 1 for further contrasts.

results further by creating targeted experimental designs that test specific hypotheses based on these findings. All posthoc $t$-tests are Bonferroni adjusted.

${ }^{20}$ Mean \pm SD. 

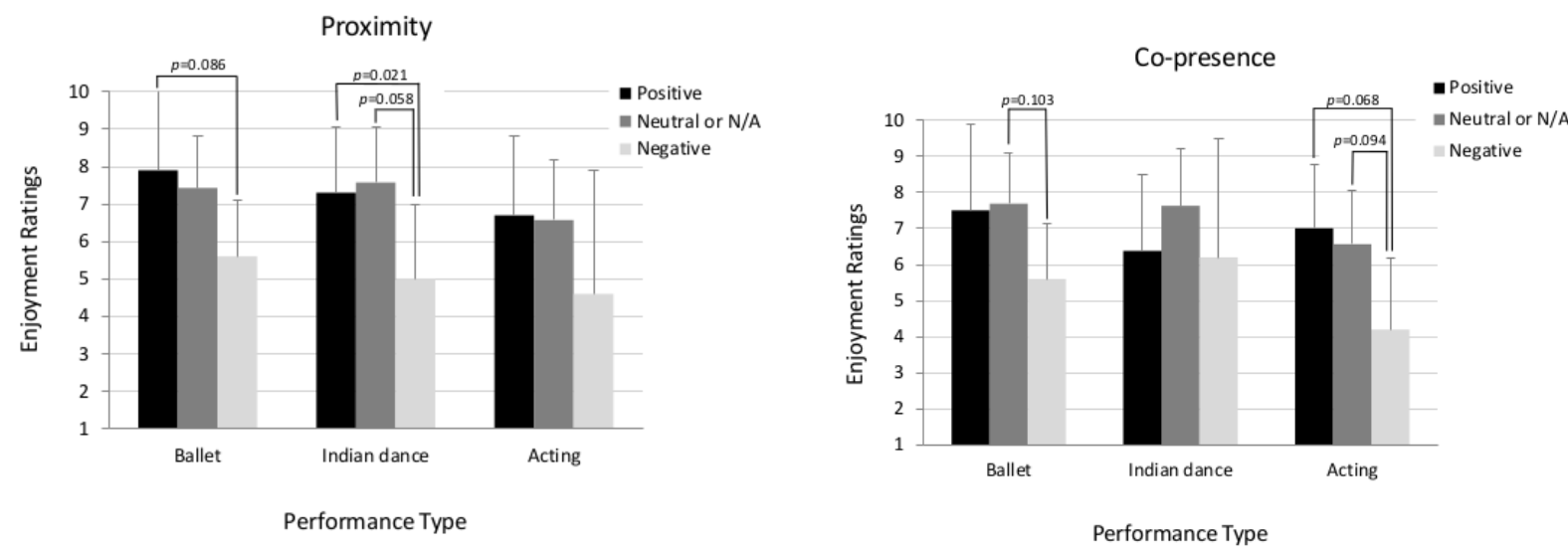

Figure 1 Effect of presence experiences on enjoyment ratings (Mean \pm SD). Left: Proximity; Right: Co-presence.

Neither tests for empathic abilities showed significant links with proximity or co-presence experiences (AQ: $p \geq 0.668$; IRI: $p \geq 0.156$ ). As we had previously found that the ability to take another person's perspective - one of the subscales of the IRI - modulated sensorimotor simulation, ${ }^{21}$ we tested the four IRI subscales ${ }^{22}$ independently. We found a strong trend toward the significant effect of co-presence ratings on perspective-taking $[\mathrm{F}(2,28)=3,052, p=0.064]$. Post-hoc $t$ -

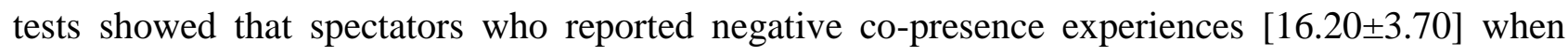
compared to those who reported neutral co-presence [20.94 \pm 3.82$]$ scored lower on perspectivetaking abilities. None of the other subscales or $t$-tests reached significance [ $p \geq 0.132]$.

\footnotetext{
${ }^{21}$ Jola et al., 'Motor Simulation Without Motor Expertise'.

${ }^{22}$ Perspective Taking (the tendency to spontaneously adopt the psychological point of view of others); Fantasy (taps respondents' tendencies to transpose themselves imaginatively into the feelings and actions of fictitious characters in books, movies, and plays); Empathic Concern (assesses 'other-oriented' feelings of sympathy and concern for unfortunate others) and Personal Distress (measures 'self-oriented' feelings of personal anxiety and unease in tense interpersonal settings. See Davies, ‘A Multidimensional Approach'.
} 


\subsection{Neuronal modification}

Descriptive values and post-hoc $t$-tests on the effects of presence experiences (positive-negativeN/A) on the spectators' performance-specific sensorimotor resonance ${ }^{23}$ in the forearm and hand muscle groups are shown in

\section{Proximity}

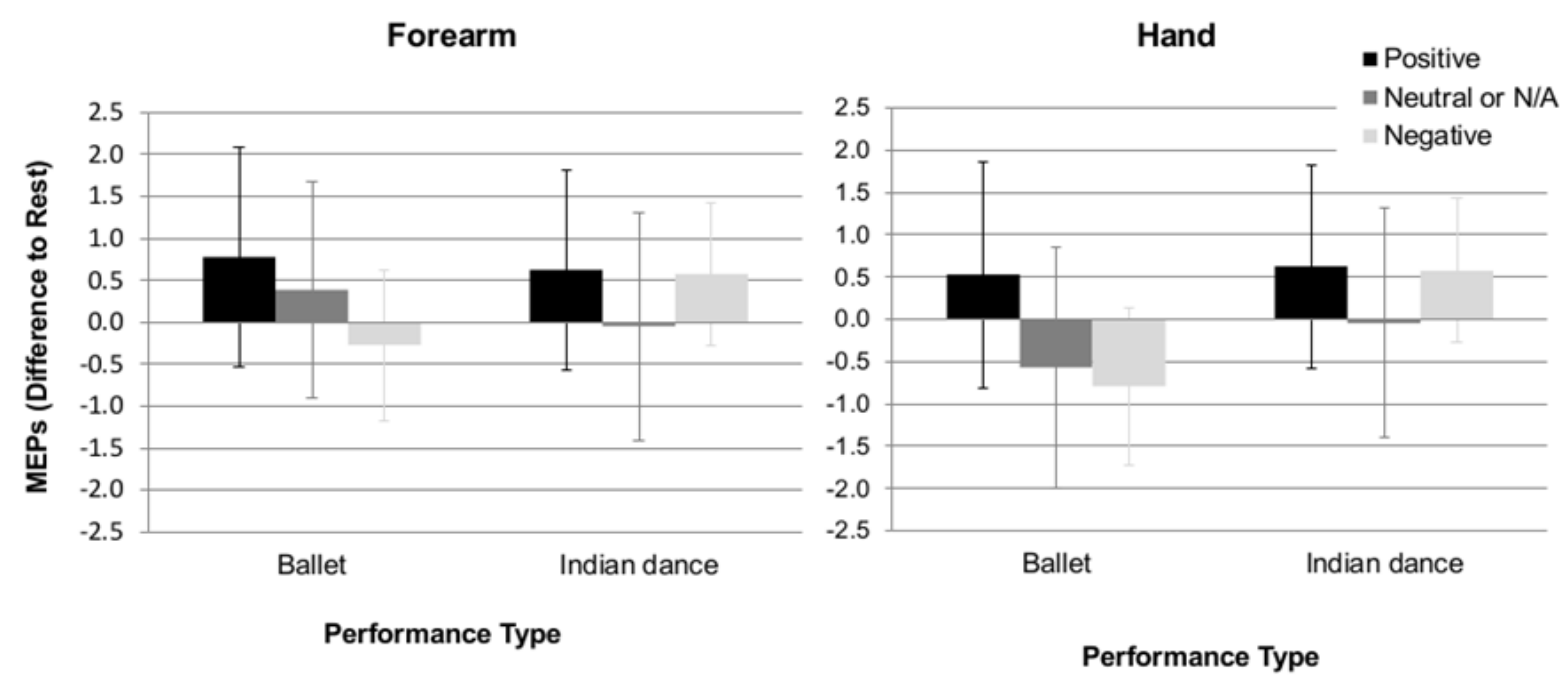

Figure 2 for proximity and Error! Reference source not found. for co-presence.

\footnotetext{
${ }^{23}$ Here, we used the same data set as in Jola et al., 'Motor Simulation Without Motor Expertise'. We therefore used the same analysis approach in exploring dance-specific MEPs gained by subtracting the acting control MEPs from the dance performance MEPs. This differs from the approach in Jola and Grosbras, 'In the Here and Now', where only the novices from the live performance were included in the analyses and where all three performance MEPs were subtracted from the rest-MEPs. According to both studies, we included age as a covariate.
} 


\section{Proximity}

Forearm

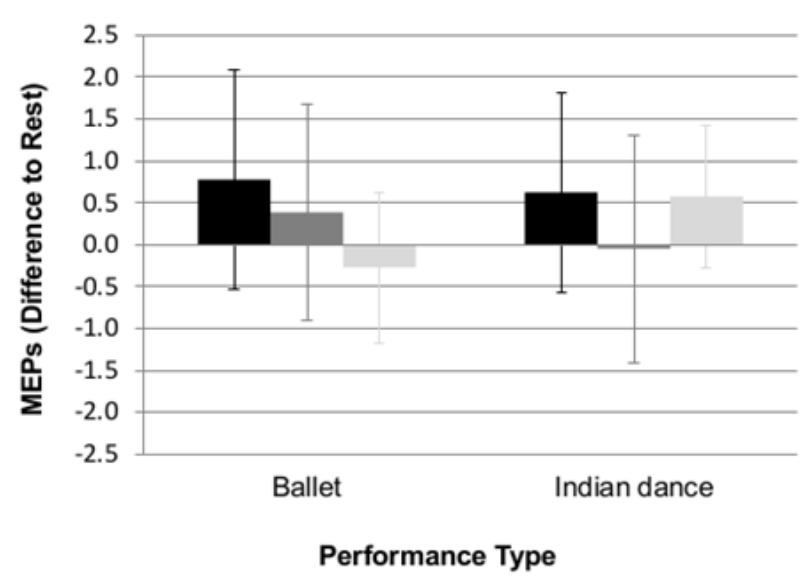

Hand

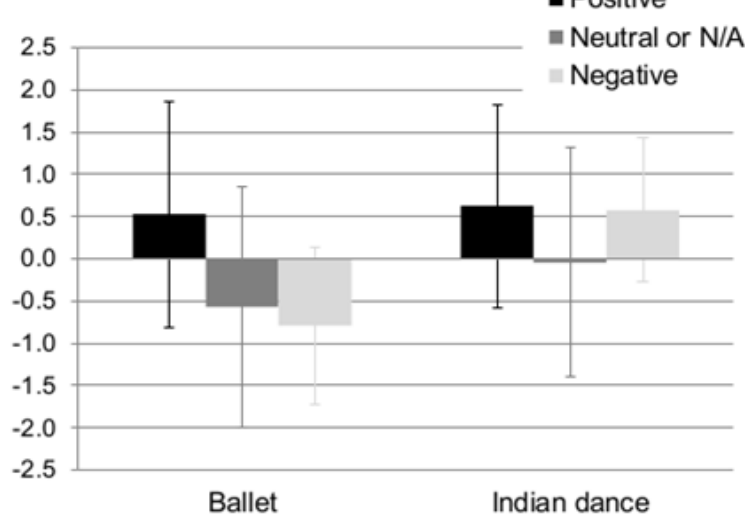

Performance Type

Figure 2. Effects of proximity on sensorimotor simulation. Mean \pm SD of MEPs in the forearm (figure left) and hand (figure right) in response to how proximity was experienced (positive-neutral-negative).

Proximity analyses showed no significant main effect [all $p \geq 0.176]$. Notably, the interaction between performance and proximity showed a trend towards a significant effect in the hand if age was not considered as a covariate factor $[\mathrm{F}(2,26)=2.682, p=0.087]$. This trend is based on the differences in proximity experience in response to watching ballet $[\mathrm{F}(2,26)=2.526, p=0.099]$ rather than in response to Indian dance-specific movements $[\mathrm{F}(2,26)=0.053, p=0.948]$. However, none of the post-hoc $t$-tests reached significance $[p \geq 0.171]$. 

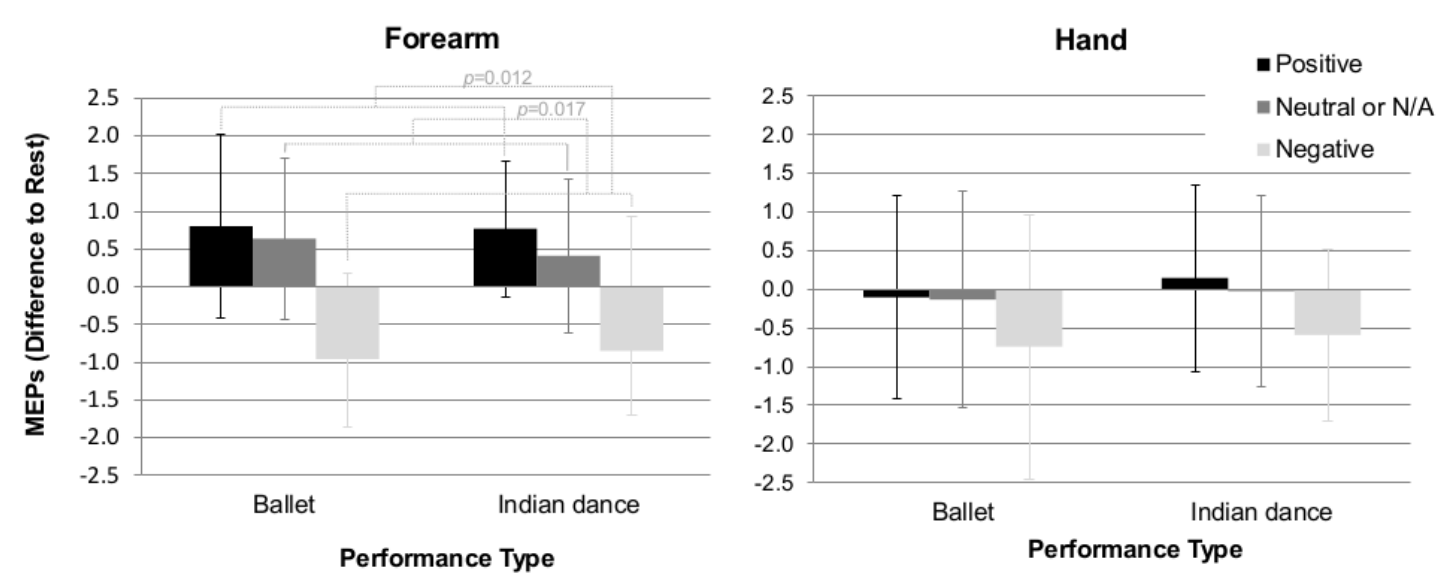

Figure 3. Effects of co-presence on sensorimotor simulation. Mean \pm SD of MEPs in the forearm (figure left) and hand (figure right) in response to how co-presence was experienced (positive-neutral-negative).

The same analysis for co-presence showed, however, a significant main effect in how it was experienced on the forearm $[\mathrm{F}(2,52)=5.700, p=0.009]$ and a strong trend toward a significant main effect of dance-specific differences on the hand $[\mathrm{F}(1,25)=2.941, p=0.099]$. All other $p$-values were 20.120. This means that co-presence experiences have a significant effect on sensorimotor resonance on the forearm in both dance-specific movements and that only hand MEPs were responsive to dance-specific performances. The post-hoc $t$-test showed that co-presence experienced negatively led to significantly reduced MEPs in the forearm [-0.96 \pm 1.13$]$ compared to co-presence experienced positively $[0.80 \pm 1,22 ; \mathrm{p}=0.012]$ or neutrally $[0.64 \pm 1.07 ; p=0.017]$.

\section{Discussion}

Presence is an elusive and far-reaching term that shifts between metaphysical, phenomenological and temporal/spatial descriptions. Importantly, however, two-thirds of our spectators noticed and 
reported presence experiences. Drawing upon some of the diverse conceptual discussions and differences in understanding the relevance of presence, we identified two connected areas proximity and co-presence - that we felt we could empirically interrogate through a combination of qualitative and quantitative methods.

We defined proximity in terms of a nearness in space, characterized as the potential ability to touch the performer (even if that ability was never acted upon). Co-presence was defined in terms of a sense of reciprocity, that both spectator and performer were 'mutually' aware of each other sharing the same time and space. Notably, proximity and co-presence as two forms of presence experiences were found to be a useful and encompassing concept here since all of the spectators' notions could be assigned to either group and they were equally often mentioned. However, we found suggestions of different relationships with both proximity and co-presence across different forms of performances and categories of spectators. These differences are further discussed below.

The qualitative interviews showed that spectators were more likely to talk about experiences of proximity than of co-presence; we also observed a tendency for spectators to report a more diverse experience of co-presence (positive and negative) than of proximity. The quantitative data further showed that spectators who had visual experience in Indian dance mentioned presence experiences in general proportionally less than experienced ballet spectators or novices. This is rather surprising, since presence in the form of proximity was often related to facial expressions that are hugely relevant in the Indian dance employed here. That presence was an important element of Indian dance can be seen in the spectators' low enjoyment ratings for this performance if they also experienced proximity negatively. We thus propose that Indian dance spectators were more familiar with forms of presence and were therefore less likely to report it.

Importantly, we found that presence experiences were significantly associated with enjoyment ratings in both proximity and co-presence experiences. This is of huge relevance since 
enjoyment was previously found to be linked with neuronal responses to watching dance. ${ }^{24}$ Here, negatively experienced proximity led to a reduced enjoyment of the Indian dance and, to a lesser extent, to a reduced enjoyment in watching ballet. Furthermore, negatively experienced co-presence was found to reduce enjoyment in watching the acting control condition and, to a minor degree, also in watching ballet. Particularly for co-presence, we found that the experience was significantly linked to sensorimotor resonance in the forearm across all dances. Positively experienced copresence and neutral experienced co-presence led to increased sensorimotor resonance when compared to negative experienced co-presence. This is in line with other studies which found that negative emotional valence reduces sensorimotor resonance in relevant muscle groups. ${ }^{25}$ In our examination, it thus seems that while sensorimotor resonance is modulated by co-presence for a variety of dance moves, proximity experiences affect sensorimotor resonance dependent on the dances. In particular, the differences in proximity are further manifested in the tendency for spectators' sensorimotor resonance when watching ballet only. Moreover, MEPs classified for proximity experiences in response to Indian dance showed a different - although not significant pattern with neutral experiences showing the smallest MEPs. This is in line with the study by Borgomaneri et al. where an increase in excitability for positive as well as negative emotional stimuli compared to neutral stimuli was found. ${ }^{26}$ In our study, another interesting difference between spectators' experiences was that experienced ballet spectators exhibited a strong tendency to report proximity more often as a positive than as a negative experience when compared to novices.

\footnotetext{
${ }^{24}$ Jola et al., 'Motor Simulation Without Motor Expertise'; Grosbras and Jola, 'In the Here and Now'.

25 Peter G. Enticott, Bronwyn A. Harrison, Sara L. Arnold, Kaitlyn Nibaldi, Rebecca A. Segrave, Bernadette M. Fitzgibbon, Hayley A. Kennedy, Kristal Lau, Paul B. Fitzgerald, 'Emotional Valence Modulates Putative Mirror Neuron Activity', Neuroscience Letters, 508(1) (2012).

${ }^{26}$ Borgomaneri et al., 'Motor Mapping of Implied Actions During Perception of Emotional Body Language.
} 
We therefore propose that different forms of performance invite or depend upon different forms of presence. Drawing on other research with dance audiences we would suggest that ballet is experienced as a visual form, whereby proximity produces a stronger visual encounter, but in comparison to other dance forms it does not rely on mutuality between performer and audience. The experience of presence and particularly co-presence is therefore connected to specific aesthetic traditions as well as to neuronal processes.

There are some limitations to this study. Firstly, only a few differences in our sample reach statistical significance levels. This is not surprising considering the small sample. Secondly, the analyses were, however, based on an existing dataset and significance levels should be corrected for multiple testing. Moreover, the original dataset explored differences in neuronal responses based on spectators' visual experience. Here we were not able to include visual expertise as an additional factor. Aspects of visual familiarity with the performances are thus not visible in this study. Finally, for comparison reasons, the sensorimotor resonance tests were based on previously conducted analyses, which looked at dance-specific movements only. This means that differences in the acting control condition were related to the dances and not treated independently. Nevertheless, we showed that spectators experienced presence in particular ways during a performance, and that differences in how they are reported are potentially linked to how audiences resonate with the observed actions. This is an important aspect for future research on action observation and social interaction.

It is also interesting to relate our findings to understandings of kinaesthetic empathy. Here we found that co-presence experiences and empathic abilities were linked. Spectators who experienced co-presence negatively scored lower on perspective taking abilities than those who experienced co-presence positively. While proximity could be characterized as involving the self looking at the other, protected by the aesthetic and psychological barrier of the forth wall, copresence entails the return of the gaze and within that the existential challenge that comes from being looked at. We propose that this can be too challenging if the spectator lacks in perspective 
taking abilities. This finding expands Jola et al., where experiencing performers' closeness was challenging for certain spectators' personalities. ${ }^{27}$ Thus, we may term a kinaesthetic response that includes a negative and hindering enjoyment, a kinesthetic dissonance characterized by uneasiness or discomfort at the reciprocal presence of the other.

Inevitably this short study perhaps raises more questions than it answers. To some degree, depending on the type of performance, we found that both proximity and co-presence affect enjoyment. We thus suggest that experiences of different forms of presence affect spectators' enjoyment of dance. While proximity, in terms of providing the spectator with the opportunity to see more and see better, might be considered less problematically enjoyable, enhancing a sense of kinesthetic empathy, the challenging experience of co-presence impacts in ways that are more unique to live experience and raises questions about the discomforting experience of empathy that is present in several types of dances.

\section{Further references:}

R. M. Conroy, 'Dance', in A. C. Ribeiro (ed.), The Continuum Companion to Aesthetics (London: Continuum International Publishing Group, 2012).

F. Hall, 'Dance Notation and Choreology', in R. Copeland, C. Marshall (eds), What Is Dance? (Oxford and New York: Oxford University Press, 1983).

\footnotetext{
${ }^{27}$ Corinne Jola, Frank E. Pollick, Beatriz Calvo-Merino, “'Some Like It Hot”: Spectators Who Score High on the Personality Trait Openness Enjoy the Excitement of Hearing Dancers Breathing Without Music', Frontiers in Human Neuroscience 8 (2014).
} 
Corinne Jola, Phil McAleer, Marie-Hélène Grosbras, Scott A. Love, Gordon Morison, Frank E.

Pollick, 'Uni- and Multisensory Brain Areas are Synchronised Across Spectators when Watching Unedited Dance', i-Perception 4 (2013).

Valerie Preston-Dunlop, Lesley-Ann Sayers, 'Gained in Translation: Recreation as Creative Practice', Dance Chronicle 34(1) (2011).

Matthew Reason, Corinne Jola, R. Kay, D. Reynolds, J.-P. Kauppi, M.-H. Grobras, J. Tohka, Frank E. Pollick, 'Spectators' Aesthetic Experience of Sound and Movement in Dance Performance', The Psychology Aesthetics and the Arts (under revision).

Matthew Reason, 'Watching Dance, Drawing the Experience and Visual Knowledge', Forum for Modern Language Studies 46(4) (2010).

The research presented in this paper was developed as part of a larger project, Watching Dance: Kinesthetic Empathy, that was funded by the Arts and Humanities Research Council, UK. 